\title{
IDENTIFICACIÓN DE HÁBITATS POTENCIALES, EFECTIVOS Y CONECTIVIDAD PARA LA FAUNA SILVESTRE, NANDAIME, NICARAGUA
}

\section{IDENTIFICATION OF EFFECTIVE POTENTIAL HABITATS, AND CONNECTIVITY FOR WILDLIFE, NANDAIME, NICARAGUA}

\author{
Miguel Garmendia Zapata ${ }^{1}$, Jurgen Guevara Alonzo ${ }^{2}$, Jorge Cisneros ${ }^{3}$ \\ ${ }^{1}$ Docente del Departamento de Manejo de Bosques y Ecosistemas, Facultad de Recursos Naturales y del Ambiente (FARENA). \\ garmendiaz@una.edu.ni \\ ${ }^{2}$ Ingeniero en Recursos Naturales y del Ambiente, Asistente SIG aplicado a Biodiversidad y Ecología de Paisaje ESTEC S.A. \\ (Enviromental System and Tecnhological Solutions).jurguevara@gmail.com \\ ${ }^{3}$ Representante legal de ESTEC S.A. (Enviromental System and Tecnhological Solutions). joroci@gmail.com
}

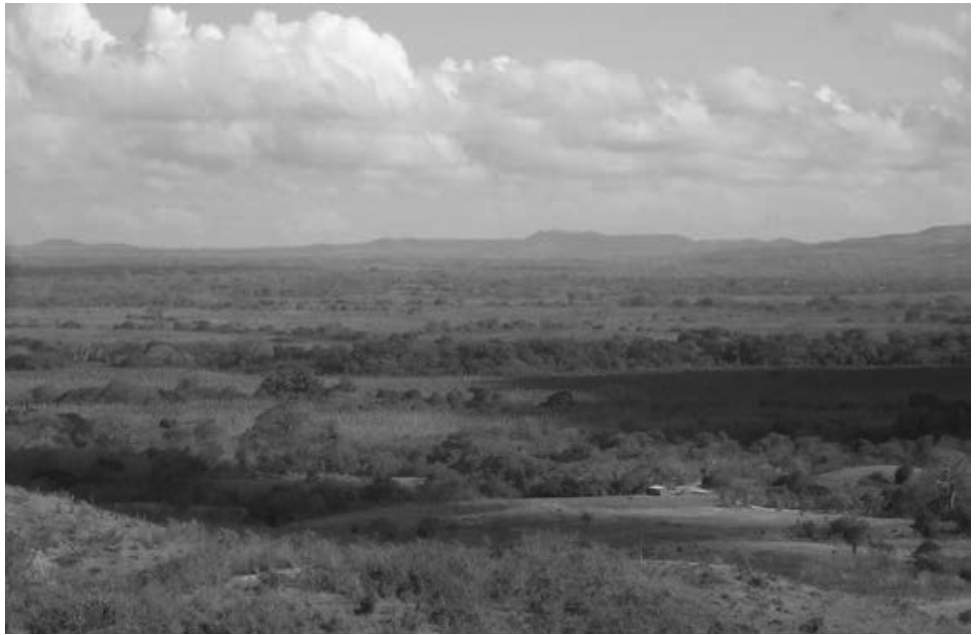

\section{RESUMEN}

El presente estudio se propone determinar los hábitats efectivos y potenciales dentro de un parche de bosque seco secundario de 4.26 ha y la existencia de conexión de ésta área con otra área natural (estudio de paisaje) que sirva de corredor a la fauna silvestre en la comunidad La Chipopa, Nandaime. Los tipos de hábitats se seleccionaron según criterios florísticos. Para el estudio de conectividad y fragmentación se realizó un análisis con imágenes de satélite para determinar las clases de cobertura de la zona y se utilizaron como firmas espectrales las clases de cobertura vegetal 2011 del Ministerio de Agropecuario y Forestal (MAGFOR), la herramienta V_LATE 1.1 y la herramienta corridor desinger. Se determinaron siete tipos de hábitat. El hábitat de mayor tamaño fue el Área de árboles con poca infección con lianas (AAPL) (3.05 ha) la cual representa el $72 \%$ del área total. En registro bibliográfico se determinaron 271 especies en el paisaje, de éstas, 102 (38\%) no encontrarían hábitat dentro del área. Los hábitats efectivos se determinaron por medio de muestreos anteriores, la mayor cantidad de especies (11 o el 33\%) se asocia con AAPL. Dentro de los hábitats potenciales se encuentran: AAPL (23 o el 17 \%) y Área de árboles con mucha infección por lianas (AAML) (12 o el 9\%), sin embargo se determinaron que 26 especies (19\%) prefieren toda el área como hábitat potencial.
This study aims to identify effective and potential habitats inside a secondary dry forest patch of 4.26 ha and the existence of connection between this area and another natural area (the study of landscape) to serve as a corridor for wildlife in the Chipopa community, Nandarola, Nandaime. The habitats types were selected based on the floristic criteria. For the study of connectivity and fragmentation analysis was performed with satellite images to determine the types of coverage area and were used as spectral signatures of vegetation classes 2011 the Ministry of Agriculture and Forestry (MAG) and it was used theV_LATE 1.1 tool and the corridordesigner tool. The habitat was the largest area of trees with low infection lianas (AAPL) (3.05 ha) which represents $72 \%$ of the total area. In the bibliographic record 271 species were identified in the landscape, of these, 102 (38\%) did not find habitat within the area. The effective habitats were determined through previous sampling, the main number of species (11 or 33\%) were associate with AAPL. Among the potential habitats are: AAPL (23 or 17\%) and area of trees with many lianas infection (AAML) (12 or 9\%), however it is determined that 26 species (19\%) prefer whole area as potential habitat. The landscape structure analysis indicates that the area is divided into 17 classes of coverage, which, the areas of secondary forests cover $48 \%$ of the total while the areas of crop and pasture cover at $34 \%$. Inside the landscape matrix the closed forest use is the most fragmented area cause the shorten distance that a wildlife species should go to 
El análisis de estructura del paisaje indica que el área se divide en 17 clases de cobertura, de éstas, las áreas de tacotal cubren el $48 \%$ del total mientras que las áreas de cultivos y pastos cubren en $34 \%$. Dentro de la matriz del paisaje el uso que se encuentra más fragmentado es el área de bosque cerrado ya que la distancia mínima que una especie de fauna silvestre debería de recorrer para encontrar esa clase es de 4,184 m, las clases de cobertura menos fragmentadas fueron las áreas agrícolas y pastos. Existe una relación entre las variables forma, dimensión fractal y proximidad en relación a los usos cultivos, pasto y bosque latifoliado cerrado. El paisaje se encuentra muy fragmentado para permitir la existencia de corredores por donde fluya la fauna silvestre.

Palabras clave: hábitat, fragmentación, conectividad, corredores.

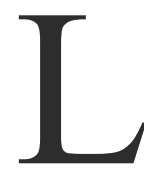

os resultados de estudios previos sobre cuantificación de diversidad biológica de fauna silvestre en la misma área de estudio fueron muy alarmantes según los monitoreos de Garmendia (2009 y 2011), la comunidad de vertebrados terrestres de la clase Mamíferos, Reptiles y Anfibios fueron poco representadas. Con intenciones de indagar en las causas, se formularon las siguientes hipótesis: puede que los hábitats dentro del área de estudio sean poco atractivos para la fauna silvestre, puede que el área de estudio sea una isla sin flujo de animales terrestres en una matriz de paisaje rodeada por áreas agrícolas y ganaderas, puede que sea la influencia humana, cazadores furtivos y caza descontrolada.

En esta investigación esta propuesto probar las dos primeras hipótesis, la hipótesis de los hábitats y la hipótesis de conectividad, la tercera hipótesis será abordada en otra investigación que tenga que ver con aspectos culturales y sociales. En la actualidad se dispone del conocimiento científico suficiente para afirmar que junto con la pérdida de hábitats, una de las principales causas de la pérdida de diversidad biológica es la fragmentación y pérdida de conectividad funcional de los espacios naturales causada por el desarrollo de infraestructuras, la expansión urbana y la intensificación agraria (Martínez, 2009).

Los paisajes, principalmente del bosque seco del pacífico de Nicaragua han sido tan atropellados por muchas años y actualmente la fragmentación se concibe como uno de los factores principales en la perdida local de especies de fauna silvestre, iniciando con los mamíferos de gran tamaño, dado a sus rangos de acción y a que son más cotizados por los cazadores. Pero hace falta mucho para poder confirmar eso desde un punto de vista meramente científico - técnico. Creemos fielmente en la ecología de paisaje como una herramienta base para la conservación de los paisajes y por ende de los hábitats del bosque tropical seco nicaragüense.

Con esta investigación se pretende sentar la línea base para continuar estudios sobre conectividad y hábitats en el bosque tropical seco de Nicaragua con el fin de impulsar proyectos de restauración y de ordenamiento territorial con el propósito de conservar y de incrementar la conectividad y la restauración de hábitats en estos tipos de ecosistemas. find other closed forest is $4.184 \mathrm{~m}$, cover classes less fragmented were agricultural areas and pasture. There is a relationship between variables shape, fractal dimension and proximity related to the uses crops, grass and broadleaf forest closed. The landscape is too fragmented to allow the existence of corridors where wildlife flows. Keywords: habitat, fragmentation, connectivity, corridors.

\section{MATERIALES Y MÉTODO}

Área de estudio. La presente investigación se llevó a cabo en la localidad del rio Medina, en la comarca la Chipopa aproximadamente a 7 kilómetros al sur de la Ciudad de Nandaime, departamento de Granada entre las coordenadas $11^{\circ} 42^{\prime} 30^{\prime \prime} \mathrm{N}$ y $86^{\circ} 05^{\prime} 30^{\prime}$ W. El área de estudio se caracteriza por tener una temperatura de entre $26^{\circ} \mathrm{C}$ y $29^{\circ} \mathrm{C}$; con un promedio anual de precipitación $1444 \mathrm{~mm}$; las máximas elevaciones se encuentran entre los $103 \mathrm{msnm}$ en las partes más bajas y los $261 \mathrm{msnm}$ predominando un relieve ondulado con pendientes menores de $30 \%$, aunque también es posible encontrar pendientes mayores de $45 \%$ en trechos muy cortos (MARENA, 1994). El tipo de vegetación existente en la zona es del tipo zonal y corresponde al bosque bajo o mediano caducifolio.

Está clasificada dentro del tipo de bosque correspondiente a tacotal, estos se forman como consecuencia del abandono de las áreas agrícolas; estos tipos de bosque se caracterizan por la aparición de especies llamadas pioneras o invasoras además de un sotobosque compuesto principalmente por especies arbustivas y algunas especies arbóreas (Ponce y Montalbán, 2005). El sitio específico en donde llevo a cabo el estudio de hábitats fue un parche de bosque secundario conocido como la Chipopa, el cual pertenece al señor Carlos Sosa, este presenta un estado sucesiones de 19 año.

Proceso metodológico. El proceso metodológico para llevar a cabo esta investigación se dividió en base a sus dos componentes, la identificación de hábitat a lo interno del parche de Nandarola y el análisis de fragmentación y diseño de los corredores a nivel del paisaje Nandarola-Mombacho.

Identificación de hábitats efectivos y potenciales. Para la identificación de hábitat primero se delimitaron los hábitats haciendo recorridos libres dentro del parche diferenciando cada tipo de hábitat en base a criterios florísticos como: densidad de lianas, dominancia de alguna especie en termino de densidad, dominancia de formaciones vegetales (áreas dominancia por herbáceas, por arbustos y/o arboles). 
Luego se procedió a realizar una caracterización de los hábitats mediante una evaluación rápida de la riqueza, abundancia y estructura (vertical y horizontal) de la vegetación de cada tipo de hábitat. Para ello se establecieron un total de 7 parcelas (una por cada tipo de hábitat identificado) distribuidas de manera aleatoria en cada hábitat. También se elaboraron diagramas de perfil para el cual se utilizaron tres transectos, distribuidos de manera que cada transepto tomara cubriera tres tipos de hábitats; las variables tomadas para ésta última actividad fueron: altura de la copa, ancho de la copa, altura de reiteración, diámetro a la altura del pecho y nombre común de la especie.

Para finalizar cada hábitat fue clasificado como efectivo y potencial, primeramente se determinaron las especies que efectivamente están en el sitio y las especies que potencialmente podrían estar en el sitio y luego éstas se vincularon a los hábitats anteriormente determinados mediante una relación demanda - oferta, en éste caso, demanda de los animales versus oferta de los hábitats. Información sobre los hábitats efectivos y potenciales se encuentra en Ojasti (2000).

Las especies efectivas fueron las especies que se determinaron en muestreos anteriores en el sitio según Garmendia (2009 y 2011) y las especies potenciales fueron determinadas mediante revisión bibliográfica, algunas de estas literaturas fueron: Neotropical Rainforest Mammal (Emmons, 1997), la Guía Ilustrada de especies de Costa Rica (Stiles\&Skuth, 1989), Anfibios y Reptiles de Nicaragua (Köhler, 2001), Plan de manejo del Volcán Masaya (Fundación Cocibolca, 2006).

A los datos resultantes se les aplicó estadística descriptiva y de inferencia. Para la estadística descriptiva se utilizaron gráficos de barra y de pastel para representar las frecuencias, totales y medias de los datos. Como medida de tendencia central se utilizó la media aritmética. Las pruebas estadísticas se realizaron con un nivel de significancia del 0.05 . Entre las pruebas estadísticas realizadas se encuentra el estadístico de la $\mathrm{X}^{2}$. La base de datos en la que organizó la información estaba estructurada con las variables en las columnas (campos) y la secuencia de toma de datos en las filas (registros). Para ello se utilizó el programa Excel $^{\circledR 2} 2010$ de Microsoft. A partir de esa base principal se derivaron todas las bases secundarias para análisis específicos con el uso de la herramienta de tablas dinámicas de Excel $^{\mathbb{}} 2010$. Luego se utilizó el programa InfoStat Versión 2010 (Di Rienzo et. al., 2010) para los análisis estadísticos.

Análisis de fragmentación y propuesta de conectividad. Para realizar el análisis de fragmentación del área de estudio se realizó un clasificación de la cobertura vegetal de la imagen del mosaico LANDSAT 2009 de 30m de resolución espacial mediante el Índice Normalizado de Diferencia de Vegetación (NDVI por sus siglas en ingles), para mejorar la clasificación de la zona se utilizaron como firmas espectrales las categorías de la capa de uso actual de suelo 2011 del MAGFOR e imágenes Google Earth de alta resolución espacial (10m), el área comprendió donde se encuentra el parche de interés hasta llegar a la Reserva Natural Volcán Mombacho; se decidió abarcar hasta esa área ya que el Volcán Mombacho es el área protegida más cercana y se deseaba conocer si existe algún tipo de conectividad entre este ecosistemas y el área de estudio.

Una vez seleccionada el área completa se procedió a realizar el cálculo de los índices de fragmentación a nivel del Paisaje Nandarola-Mombacho. La finalidad de los índices de paisajes es determinar el nivel de fragmentación de las unidades territoriales que componen el mosaico de vegetación dentro del área de estudio. El procedimiento de cálculo se aplicó mediante las herramientas SIG VLATE y PATCH ANALIST ambas diseñadas para el software Arc Gis versión 9.3. Los índices calculados con la herramienta VLATE fueron los siguientes: Área, borde, forma, dimensión fractal, proximidad (fragstat), áreas núcleo (búfer 100 m).

Diseño de los corredores. Después de haber generado los índices de paisaje y los polígonos de áreas núcleo se procedió realizar el análisis de los corredores el cual evalúa la utilidad de dos bloques de hábitat conectados entre sí para la movilidad de las especies potenciales; la herramienta utilizada para realizar este cálculo es el CORRIDOR DESIGNER. Esta herramienta de análisis identifica la ruta a través del corredor que minimiza la distancia entre los parches de hábitat de alta calidad.

Análisis estadístico. Los análisis estadísticos incurrieron en los cálculos de la estadística descriptiva e inferencial. La estadística descriptiva comprendió en la utilización de tablas y graficas de pastel en donde se representan las frecuencias, totales y medias; como medida de tendencia se utilizó la media aritmética. Adicionalmente se utilizó como análisis descriptivo multivariado exploratorio el análisis de componentes principales y análisis de conglomerados (Di Rienzo, 2008). Las pruebas estadísticas se realizaron con un nivel de confianza del 0.05 , entre los análisis realizados se encuentra el análisis de varianza ANOVA combinada con pruebas de comparación múltiple de Duncan para comparar las medias entre los conjuntos de datos, para explorar la normalidad entre los datos se utilizó la prueba de Normalidad de Shapiro Wilks, los datos que no cumplen con el supuesto fueron normalizados con logaritmo neperiano (ln) o analizados con pruebas homologas para datos no paramétricos.

\section{RESULTADOS Y DISCUSIÓN}

Descripción de los hábitats en base a criterios florísticos. En el área de estudio se determinaron siete tipos de hábitat, estos se diferenciaron en base a criterios florísticos y en base a las especies más representativas de cada uno. A continuación se describen cada uno. 
Área de cornizuelos (ACO). En este hábitat se identificó, gran cantidad de individuos de Acacia collinsii a esto se debe su nombre, el cual alberga gran cantidad de aves tales Campylorhynchus rufinucha, Hirundo rustica, Archilochus colubris etc. Utilizando dichas especies la parte superior de los cornizuelos para anidar, dicho área se caracteriza también por presentar en su interior riqueza de árboles de mayor edad y tamaño que ofrecen hábitat a especies efectivas como la Calocitta formosas, ocupando este área 0.073 ha el cual corresponde a un 2\% del área total (figura 1, P 1.1).

Área de árboles con poca infección con lianas (AABPIL). Conocido como el mar, nombrado de tal modo que este tipo de hábitat es el que ha tenido mayor presencia o mayor número de repeticiones en toda el área de estudio o parche de bosque y se caracteriza por contener arboles con menor presencia de lianas. Observándose en este hábitat especies efectivas de mamíferos pequeños (Sciurus vulgaris), aves (Calocitta formosas), reptiles (Ameiva quadrilineata), y anfibios (Bufo bufo) Siendo este área de mayor tamaño (3.05 ha) el cual representa el $72 \%$ del área total (figura 1, P 1.2).
Área del claro (ACL). Aquí se destaca el componente herbáceo, con menor presencia de componente arbustivo donde la mayoría de las especies herbáceas encontradas son lianas y bejucos entre ellas: Ceratophytum sp, Bytneria aculeata, Cydista diversifolia, el cual ocupa un área correspondiente a 0.048 ha, representado el $1 \%$ del área de estudio (figura 1, P 1.3).

Área de lianas en arbustos con vegetación herbácea (ALACH). En este mosaico existe gran riqueza de vegetación ya que logramos identificar diversas especies de lianas y bejucos en su mayoría en arbustos además de presentar componente herbáceo, ocupando este mosaico un área de 0.373 ha correspondiente al 9\% respectivamente. Este hábitat es efectivo para aves efectivas como Eumomota superciliosa (figura 2, P 2.1).

Área de campanitas (ACAMP). Este hábitat se caracteriza por contener en su mayoría componente herbáceo encontrándose una especie abundante siendo dicha especie: Ipomoeatrifida,presentándose un claro por carecer de árboles y arbustos. Además se identifica por ser el más pequeño de todos los hábitat siendo este de un área de 0.018 (figura 2, $\mathrm{P}$ 2.2).

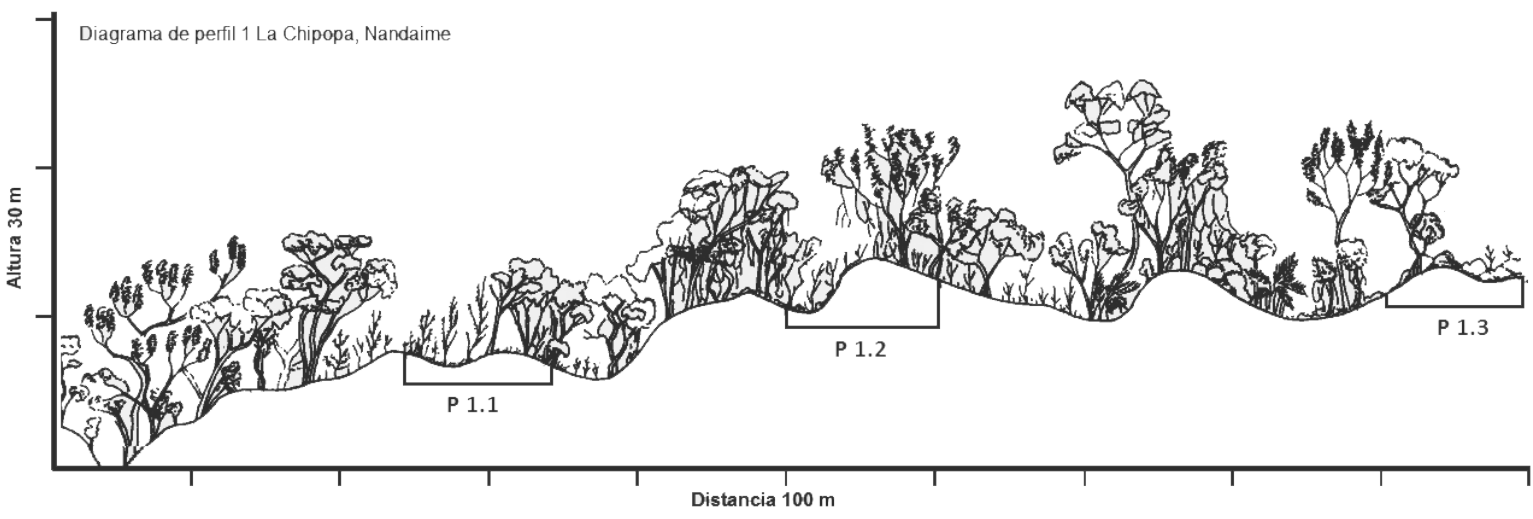

Figura 1. Diagrama de perfil 1, ( $\mathrm{P}=$ parcelas anidadas) La Chipopa, Nandaime 2012.

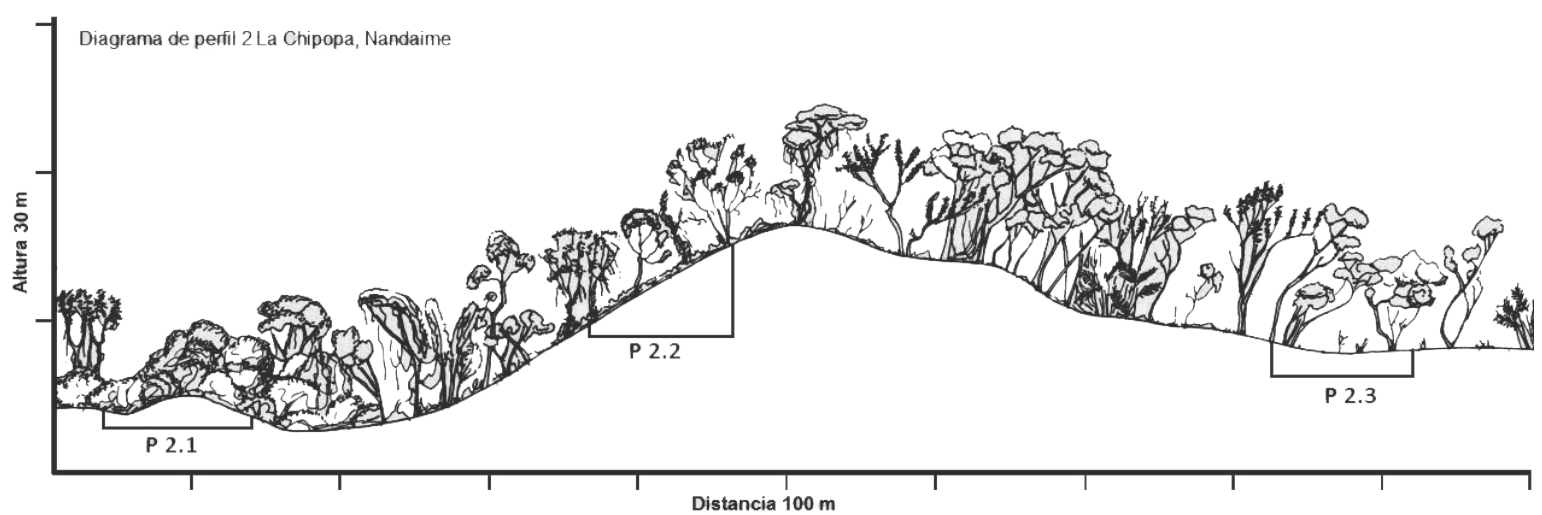

Figura 2. Diagrama de perfil 2 ( $\mathrm{P}=$ parcelas anidadas $)$ La Chipopa, Nandaime 2012. 
Área de árboles con mucha infección por lianas (AABMIL). Este tipo de hábitat se caracteriza en su mayoría por poseer árboles y arbustos con abundante presencia de lianas, el cual es un hábitat que puede albergar gran cantidad de mamíferos medianos, aves, reptiles y anfibios. Posee un área de 0.365 ha el cual representa un $9 \%$ del área de estudio (figura 3, P 3.2).

Área de lianas en arbustos sin vegetación herbácea (ALASH). En este hábitat encontramos gran presencia de lianas en el componente arbustivo, macollas de palmas entre estas Coyolito (Bactrissp). No se identificó presencia de vegetación herbácea. Representa un $8 \%$ del sitio de estudio, ocupando un área de 0.331 ha (figura 3, P 3.3).

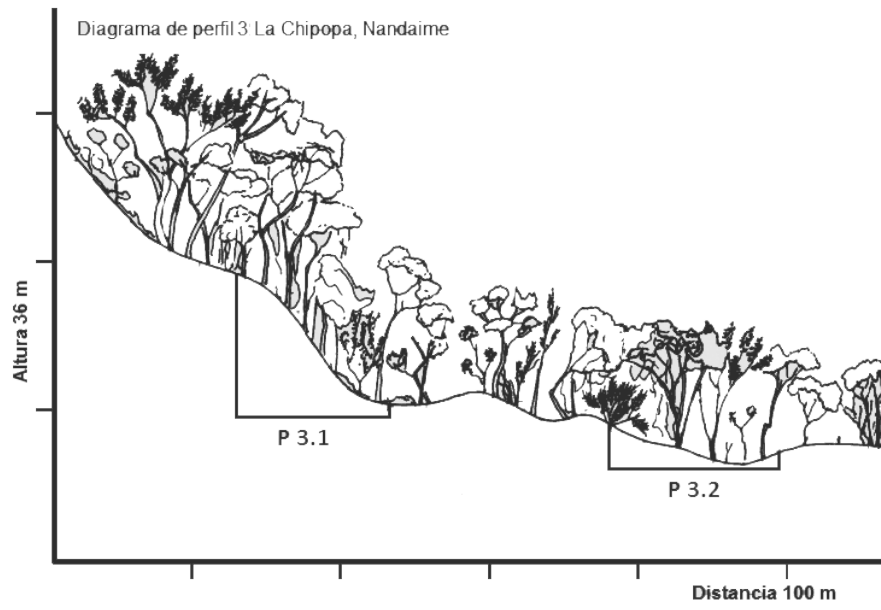

Figura 3. Diagrama de perfil 3 ( $\mathrm{P}=$ parcelas anidadas). La Chipopa, Nandaime 2012.

Composición Florística. En las parcelas establecidas se contaron 510 individuos, clasificados en 30 familias y 46 especies, de estas 25 son árboles, algunos de estos eran: Chomelia speciosa, Genipa americana, Lonchocarpus minimiflorus, 3 son arbustos, los cuales eran Combretum farinosum, Adelia barbinervis, Aphelandra scabra, 8 son bejucos, entre los más abundantes eran: Lygodium venustum, Smilax spinosa, Amphilophium sp, 9 son hierbas entre las más abundantes estaban Panicum trichoides, Selaginella mesenterica, Ipomoea trifida y una especie de palma que es un Bactris sp.

\section{Identificación de hábitats efectivos y potenciales}

Mediante revisión bibliográfica de estudios realizados en el trópico seco del pacífico se determinaron que en la zona deberían de habitar 270 especies, en éstas se incluyen las especies que se encuentran en la Reserva Natural del Volcán Mombacho. De éstas, 59 especies, que representan el $22 \%$ no encontraría lugar en el área de estudio dentro de las cuales tenemos, de esos 31(52.5\%) son propias de los ecosistemas de la Reserva Natural Volcán Mombacho (bosque nuboso)

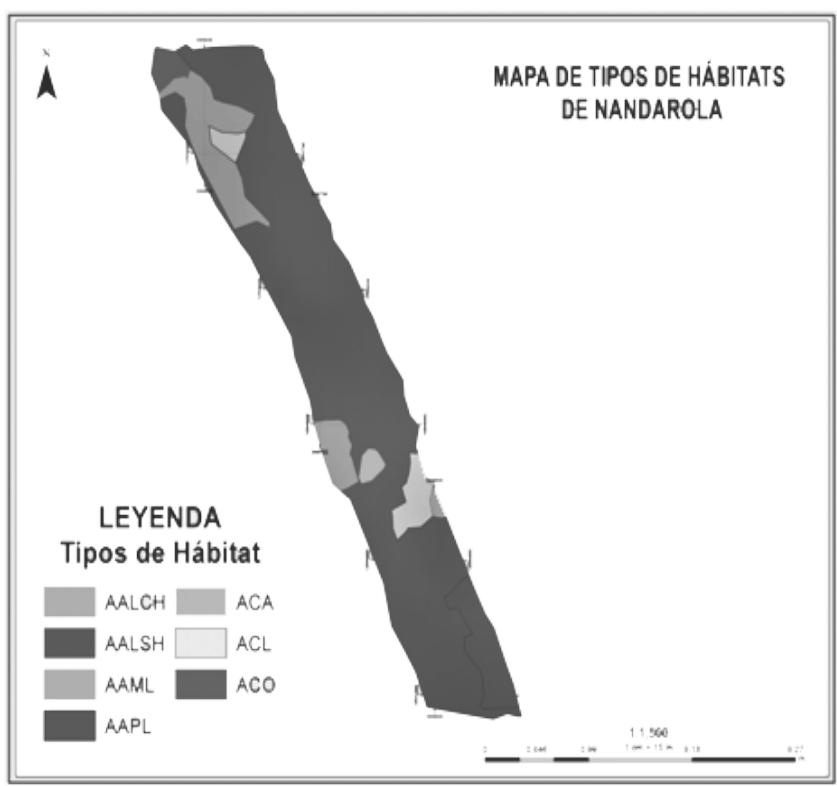

Figura 4. Mapa de los 7 tipos de hábitats de Nandarola, Nandaime, 2012.

por ejemplo: Bolitoglossa mombachoensis, Ciccaba nigrolineata, Mustela frenata, Norops biporcatus entre otras. El $20(34 \%)$ requiere de cuerpos de agua y el 8 (13.5\%) requiere la presencia de bosque de galería dentro del área. El resto de las especies eran efectivas o potenciales dentro del área de estudio.

De los siete hábitats determinados 74 (35 \%) especies son específicas de un tipo de hábitat, 70 (33\%) prefieren la combinación de más de dos tipos de hábitat y 67 correspondiente al (32\%) fueron ubicadas en todos los tipos de hábitats.

Hábitats efectivos $y$ potenciales para especies que prefieren un solo tipo de hábitat. El hábitat efectivo de las especies que prefieren un solo tipo de hábitat (18 especies) es: AABPIL (en donde se han determinado 12 especies correspondiente al $67 \%$ ), con diferencias significativas comparado a las cantidades de especies asociadas al resto de los hábitats $\left(\mathrm{X}^{2}=19.07, \mathrm{p}<0.0008\right)$, esos últimos tuvieron de 1 a 2 especies asociadas. 
Los hábitat potenciales de las especies que prefieren un solo tipo de hábitat (54 especies) son: AABPIL (en donde se han determinado 25 especies que corresponden al 46 $\%$ ) y AABMIL (en donde se han determinado 12 especies, correspondientes al $22 \%$ ), con diferencias significativas comparado a las cantidades de especies asociadas al resto de los hábitats $\left(X^{2}=12.79, p<0.0051\right)$, en esos últimosestaban asociadas de 7 a 10 especies.

Hábitats efectivos y potenciales para especies que prefieren dos o más tipos de hábitat. Los hábitats efectivos de las especies que prefieren dos o tres tipos hábitats son las combinaciones siguientes: 1. AABMIL-AABPIL, 2. AABMIL-ACAMP-ALARCH, 3. AABMIL-ACLALARCH, 4. AABMIL-ACO-ALARCH, 5. AABPIL-ACL, 6. ACAMP-ACL-ALARCH Y 7. ACL-ALARCH. En los cuales efectivamente solo se determinaron una especie para cada combinación, exceptuando la combinación AABMILACO-ALARCH donde se determinaron 2 especies, por lo tanto la cantidad de especies entre las combinaciones no varió significativamente $\left(X^{2}=0.64, p>1\right)$.

Los hábitats potenciales de las especies que prefieren dos o tres tipos hábitats son las combinaciones siguientes: 1 . AABMIL-AABPIL-ALARCH (8 especies correspondiente al $13 \%), 2$.AABPIL-ACL (7 especies que corresponden al $11 \%$ ), 3. AABMIL-AABPIL (6 especies asociadas, representando $10 \%$ ) y 4 . ACL-ACAMP (5 especies que respectivamente corresponden al $8 \%$ ), principalmente. Para el resto de las 19 combinaciones solo se asociaban de 1 a 4 especies en cada una, siendo esta asociación significativamente menor a las cuatros mencionadas anteriormente $\left(X^{2}=29.56, p<0.042\right)$.

\section{Análisis de fragmentación del paisaje Nandarola y diseño de corredores}

A continuación se describirán los resultados del cálculo de las métricas de paisaje para determinar el grado de fragmentación del paisaje así como también realizar el diseño de los corredores que permitirán un mejor flujo de especies entre el parche de intereses (Nandarola) y las áreas protegidas más cercanas a este.

Métrica de área. El paisaje Nandarola-Mombacho se encuentra dividido en 17 clases de uso, estructuralmente la clase de cobertura más predominante dentro del paisaje son los tacotales, los cuales cubren aproximadamente el $48 \%$ del total del área $(314,937.69 \mathrm{ha})$ seguida de la clase de cultivos/ pasto las cuales cubren el 34\% del área total (220,367.19 ha), posteriormente le siguen las áreas de musáceas las cuales cubren el 5\% del total del paisaje $(30,624.22$ ha) y por ultimo las áreas de cultivos anuales que cubren alrededor del $4 \%$ del total del área (29,048.85 ha). El resto de los usos dentro del paisaje comparten entre el 3 y el $1 \%$. mayor cantidad de parches, cabe mencionar que las áreas de vegetación arbustivas poseen una mayor cantidad de parches (1,218 parches); en segundo lugar se encuentra la clase de matorrales/maleza esta posee un total de 743 parches. A pesar de que estas clases poseen la mayor cantidad de parches dentro del paisaje apenas representan entre el 3 y el $5 \%$ del área total esto se debe a que los parches de vegetación de estas clases son demasiado pequeños para tener representatividad dentro de la estructura del paisaje.

Tabla 1. Clases de cobertura con mayor número de parches

\begin{tabular}{lc}
\hline Clases de cobertura & $\mathrm{N}^{\circ}$ de parches \\
\hline Cultivos anuales & 418 \\
Cultivos/pastos & 809 \\
Matorrales/malezas & 743 \\
Vegetación arbustiva & 1218
\end{tabular}

Los resultados de área y cantidad de parches por cada tipo de cobertura nos indican que dentro de la matriz del paisaje las áreas de bosque (principalmente cerrado) se encuentran muy escasos, esto supondría que dentro del paisaje las especies de fauna silvestre no tendrían muchas opciones para la movilidad de un parche a otro, ni siquiera la clase que cuenta con mayor número de parches (Vegetación Arbustiva) no cuenta con área suficiente en sus parches ya que esta representa menos del $1 \%$ en la matriz del paisaje. Esto se puede apreciar también el mapa ya que este muestra que la mayor parte del paisaje está dominado por una combinación de cultivos y pastos más áreas de tacotales.

Métrica de forma. De acuerdo al análisis de forma del paisaje se determinó que la clase que posee la forma más regular es la de bosque latifoliado abierto con un promedio de forma de 1.24 (tabla 2), seguida de las áreas de manglar y bosque latifoliado cerrado ambos con un promedio de $1.29 \mathrm{y}$ por último se encuentra las áreas de Musáceas con promedio de 1.30, cabe mencionar que las áreas de tacotal ocupa el segundo lugar en cuanto a las formas más irregulares (1.56 en promedio) la clase que posee la forma más irregular dentro del paisaje es el área de arroz bajo riego (2.05) aunque esta no es la clase más relevante dentro del paisaje.

Tabla 2. Clases de cobertura con menor índice de forma

\begin{tabular}{lrc}
\hline Clase de cobertura & NP & Promedio Shape Idx \\
\hline Musaceas & 3 & 1.30 \\
Bosque latifoliado cerrado & 341 & 1.29 \\
Manglar & 5 & 1.29 \\
Bosque latifoliado abierto & 4 & 1.24 \\
\hline
\end{tabular}


Un aspecto muy importante es que las clases del paisaje que presentan formas relativamente muy irregulares son además las que ocupan la mayor área dentro del ensamble estructurar del paisaje (cultivos/pastos y Tacotal) adicionalmente las clases de vegetación que podrían albergar especies (como las áreas de bosque cerrado y abierto) poseen las formas más regulares pero no se encuentran en gran cantidad dentro del paisaje.

En cuanto al resumen estadístico se puede notar que existe una variabilidad alta entre los datos de forma para cada clase aunque esta no sea tan marcada como para el caso de la variable áreas; la clase de cobertura que posee el mayor coeficiente de variación dentro del conjunto de datos es cultivos/pastos $(80.53 \%)$ seguida por la clase de tacotal (73.32\%) y por último la clase de bosque latifoliado cerrado $(56.03 \%)$.

Los datos obtenido por la métrica de forma del paisaje nos muestra un tendencia muy marcada a la disminución de los parches de vegetación con formas que se podrían considerar como deseables (parches con índices cercanos a 1), esto se observa principalmente en las áreas de bosque abierto y cerrado, esto además es señal de una degradación bien marcada de las áreas de bosque dentro del paisaje en estudio y por ende repercutiría directamente en las especies de fauna silvestre, ya que estructuralmente los parches de vegetación remanentes no serían capaces de sostener más especies (hablando en el caso de los mamíferos grandes y medianos) los cuales tenderían a desaparecer, o a dificultar un flujo de éstos por el paisaje .

Índice de dimensión fractal. De acuerdo a los valores del índice de Dimensión Fractal se puede observar que, al igual que el índice de forma, la clase que posee los fractales más regulares son las áreas de bosque latifoliado (1.25) principalmente las de vegetación más cerrada, seguida de las áreas de Manglar (1.28). Cabe mencionar que las áreas de tacotal y las áreas de cultivos/pastos presentan altos valores (1.29 y 1.34 respectivamente) sin embargo estas no son las áreas que poseen la mayor irregularidad en sus fractales, la clase Bosque Latifoliado Abierto es la que posee la mayor cantidad de irregularidad ya que ha alcanzado un valor de 1.35, así como también la clase Caña de Azúcar la cual presenta valores iguales a la clase anterior.

Un aspecto muy importante que es que el índice de dimensión Fractal ha dado resultado muy similares a los arrojados por el índice de forma es decir aquí se muestra como clases de gran irregularidad en la forma de sus parches las áreas de cultivos/pasto y Tacotal aunque en este sentido el índice nos muestra un comportamiento diferente cuando se trata de la clase bosque abierto la cual presenta valores más elevados que el índice anterior.

De acuerdo con los valores de dimensión fractal estos concuerdan con los resultados de índice de forma, y como era de esperarse los parches de vegetación más conservada se han remplazado por parches que estructuralmente no son capaces de albergar especies de fauna, estas limitaciones estructurales serán más sentidas principalmente por las especies de mamíferos medianos y grandes los cuales necesitan grandes área de bosque y con estructuras compactas.

Métrica de borde. En cuanto a la métrica de borde es de notar que la clase de vegetación que posee la mayor cantidad de borde promedio son las áreas de tacotal, seguida de las áreas de café con sombra; las áreas de vegetación que poseen menor cantidad de borde entre sus parches son las áreas de bosque latifoliado tanto cerrado como abierto cabe mencionar que de estas clases de vegetación la más dominante del paisajes (Tacotal) es también la que posee mayor borde promedio sin embargo no representa el mayor borde, esta categoría la ocupa la clase arroz bajo riego y caña de azúcar.

El análisis de borde nos muestra que las áreas de vegetación más cerrada son las que presentan los bordes relativamente más bajos, esto se corrobora con la información de forma de cada categoría, por otra parte las áreas de tacotales muestran los bordes más amplios esto nos indica que estas formas a pesar de ser una de las categorías más abundantes (en cuestiones de áreas y número de parches) no son capaces de albergar especies de fauna además estas categorías también presentan las formas más irregulares dentro del paisaje, es posible que talves está formas estructurales puedan ser usadas por las especies como trampolín y no como un área definitiva, es posible que a través de diferentes parches de tacotal puedan llegar especies hacia el parche de estudio.

\section{Análisis de fragmentación del paisaje}

A continuación se describe el análisis de fragmentación en base al índice de proximidad y el modelo de regresión múltiple, el cual integra todos los índices de paisaje y la métrica de áreas núcleo.

Índice de proximidad del paisaje. El análisis de fragmentación fue el resultado del cálculo del índice de proximidad obtenido para todas las clases dentro del paisaje, para las clases de vegetación se obtuvo como resultado que las áreas de tacotal se encuentran separadas por distancias no mayores a $300 \mathrm{~m}$ entre sí, esto hace suponer que esta clase podría ser alcanzada por especies de desplazamiento relativamente largo mas no por especies que sus rangos de acción son relativamente cortos.

En el caso de las áreas de bosque latifoliado cerrado el índice nos muestra que esta clase de vegetación es la que se encuentra más fragmentada ya que la distancia mínima que una especie debería de recorrer para alcanzar un parche de la misma categoría es de $4 \mathrm{~km}$ (4,184 metros) esto hace suponer que esta clase no podría ser alcanzada por especies cuyos rangos de movilidad sean inferiores a este rango por 
ende este clase es la que se encuentra más fragmentada dentro del paisaje.

Análisis de áreas núcleos. De acuerdo con el análisis de los núcleos de densidad de las clases de cobertura del paisaje se ha encontrado que la clase que posee mayor núcleo dentro de sus parches es el café con sombra (más de 300000 metros) seguida de la clase de bosque latifoliado cerrado y por último la clase de tacotales se encuentra en tercer lugar en cuanto a densidad en sus parches.

A pesar de que la clase de bosque cerrado alcanzo valores aceptables en cuanto a los núcleos de densidad de sus parches, esta clase se encuentra demasiado aislada en el paisaje para ser de utilidad por alguna de las especies de fauna registradas además de esto es de notar también que esta categoría se encuentra dentro del área núcleo de la Reserva Natural Volcán Mombacho.

Diseño de los corredores. El diseño de los corredores se realizó tomando en cuenta solamente las clases de vegetación que pudieran servir como trampolín para las especies y las que tuvieran las formas estructurales más regulares y las más cercanas dentro del paisaje; así pues se determinó que el 54.6\% del área de los corredores se encuentra en la clase tacotal ya que esta clase posee una distancia no mayor de 300 metros entre clase y clase y es puede ser alcanzada por las especies, en segundo lugar se encuentra la clase de Matorrales/Maleza con un $17.9 \%$ del total y por último en tercer lugar se encuentra la clase de vegetación arbustiva con un $11.9 \%$ del total, cabe mencionar que se tomó un pequeña porción de áreas de cultivos y pastos pero estas representan menos del 1\% (tabla 3). A pesar de que las clases de tacotal y matorrales poseen áreas relativamente grandes estadísticamente las diferencias entre las clases en cuanto al área resultaron no ser tan significativas $(\mathrm{F}=2.28, \mathrm{P}<0.0415)$ porque realmente no existe diferencia entre las áreas de los clases que pertenecen a cada corredor.

Tabla 3. Área de los corredores por clase de cobertura

\begin{tabular}{lcc}
\hline Clases de cobertura & Área (ha) & Área (\%) \\
\hline Bosque latifioliado cerrado & 1743 & 7.2 \\
Café con sombra & 1623 & 6.7 \\
Cultivos/pastos & 199 & 0.8 \\
Matorrales/malezas & 4312 & 17.9 \\
Tacotales & 13177 & 54.6 \\
Vegetación arbustiva & 2721 & 11.3 \\
Vegetación herbácea & 370 & 1.5 \\
\hline Total & 24146 & 100.0 \\
\hline
\end{tabular}

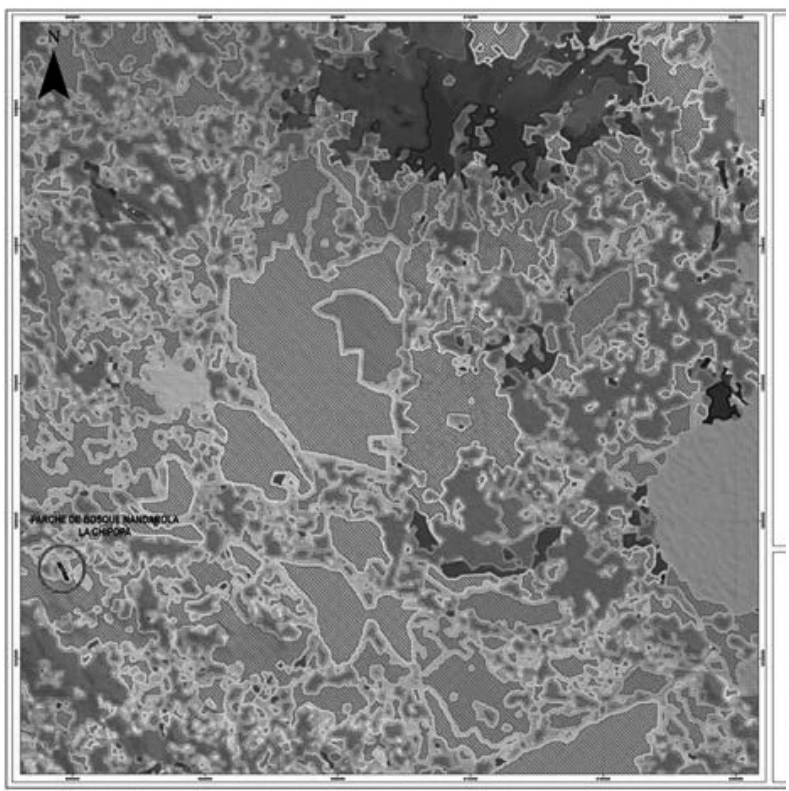

Figura 5. Mapa de conectividad del paisaje Nandarola-Mombacho.

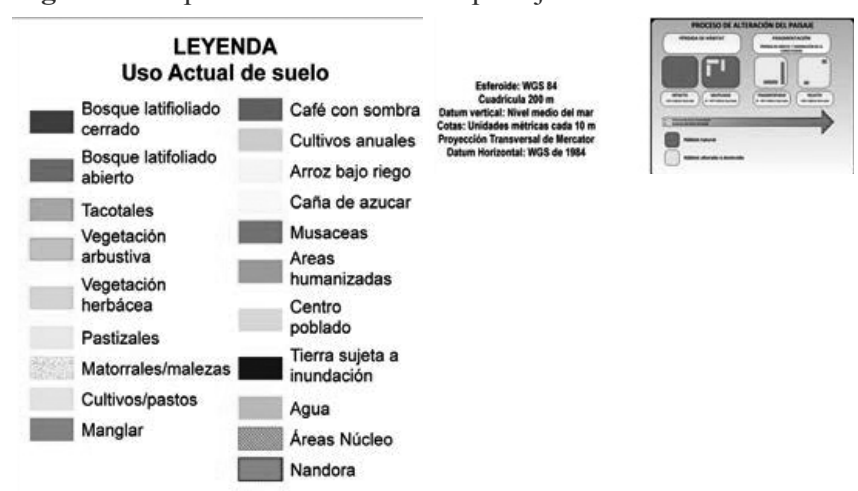

CONCLUSIONES

En el área de estudio se identificaron siete tipos de hábitat dentro del mosaico del parche de bosque en la comunidad La Chipopa, Nandaime, estos fueron: área de árboles con muchas lianas (AAML), área de árboles con pocas lianas (AAPL), área de arbustos con lianas y sin vegetación herbácea (AALSH), y área de arbustos con lianas y vegetación herbácea (AALCH), área de cornizuelos (ACO), área del claro (ACL), área de campanitas (ACA).

Los hábitats referidos al área de árboles con poca por lianas (AAPL) y el área de mucha con lianas (AAML) y en menor medida el área de arbustos con lianas y vegetación herbácea (AALCH) son los hábitats efectivos y potenciales dentro del área de estudio.

A pesar de poder formarse corredores, la alta fragmentación del paisaje hace imposible que las áreas de corredores puedan alcanzar el parche en la Chipopa, Nandarola lo que significa que éste se encuentra aislado y no podría ser alcanzada por las especies potenciales provenientes de otras áreas cercanas. 


\section{REFERENCIAS BIBLIOGRÁFICAS}

Castro G; Tigabu, M, Odén, PC. 2009. A Chronosequence analysis of forest recovery on abandoned agricultural field in Nicaragua. Journal of Forestry Research. 20(3): 213-222.

Di Rienzo, JA, Casanoves, F; Balzarini, MG; González, L; Tablada, M, Robledo, CW. InfoStat versión 2010. Grupo InfoStat, FCA, Universidad Nacional de Córdoba, Argentina. URL http://www.infostat.com.ar

Emmons, L. 1997. Neotropical Rainforest Mammals. A field guide. 2 ed. Chicago US. 307 p.

Fundación Nicaragüense para la Conservación (Fundación Cocibolca) 2006. Plan de Manejo de la Reserva Natural Volcán Mombacho, $136-142 \mathrm{p}$.

Garmendia Z, M. 2009. Diversidad de Fauna Silvestre en Bosque Seco Tropical, comunidad La Chipopa, Nandaime, Nicaragua. Publicado en la revista científica La Calera Año 9, No 12, 12-20.

Garmendia Z, M. 2011. Monitoreo de la Fauna Silvestre en Bosque Tropical Seco Secundario, Nandarola, Nandaime, Nicaragua. Publicado en la revista científica La Calera Año 11 No 16, 24-32.

Gonzales, B. 2005. Tree species Diversity and Regeneration of Tropical Dry forest in Nicaragua. Ph.D. Thesis. Swedish University of Agricultural science. 2 p.

Harcourt C.S and Sayer J.A. 1996. The conservation atlas of tropical forest.TheAmericas. Simon\&Schuster.

Instituto nacional de biodiversidad, Inbio (en línea), consultado 31 marzo 2012, disponible en http://www.inbio.org.pi/.

Janzen, DH. 1988. Management of habitat fragment in a tropical dry forest: growth. Annals of Missouri Botanical Garden 75: $105-116$.

Köhler, G. 2001. Anfibios y Reptiles de Nicaragua. Editorial Herpetonoffbach. Alemania, DE. 208 p.

Loh, J; Wackernagel, M. (Eds) 2004. Informe Planeta Vivo, 2004. WWF, Gland.

MARENA (Ministerio del Ambiente y los Recursos Naturales, NI).1994. Proyecto Nandarola/ MARENA- DED, Plan de manejo de la cooperativa Pedro Joaquín Chamorro Cardenal. Proyecto de protección del bosque. Managua, Nicaragua. 35p.

Martínez A, C; Múgica de la Guerra, M; Castell P, C; De Lucio F, J. 2009. Conectividad ecológica y áreas protegidas. Herramientas y casos prácticos. Ed. FUNGOBE. Madrid. 86 p.

McGarigal, K; Cushman, SA; Neel, MC; Ene, E. 2002. FRAGSTATS: Spatial Pattern Analysis Program for Categorical Maps. Computer software program produced by the authors at the University of Massachusetts, Amherst. Available at the following web site: http:// www.umass.edu/landeco/research/fragstats/fragstats.html.

Moreno C, E. 2001. Métodos para medir la Biodiversidad. M\&T-Manuales y tesis SEA. Ed. CYTED (Programa Iberoamericano de ciencia y tecnología para el desarrollo). Vol.1. Zaragoza, ES. 84 p.

Ojasti, J. 2000. Manejo de Fauna Silvestre Neotropical. Ed. F, Dallmeier. Washington. 281 p.

Pérez, A. 2004. Aspectos conceptuales, análisis numérico, monitoreo y publicaciones del datos sobre biodiversidad. MARENA. Centro de Malacología/Diversidad animal UCA. Managua, Nicaragua. $331 \mathrm{p}$.

Roldan, H. 2001. Recursos forestales y cambio de uso de la tierra, República de Nicaragua. Santiago, Chile. 73 p.

Sabogal, C, Valerio, L. 1998. Forestcomposition, structure and regeneration in a dryforest of the Nicaraguan Pacific coast. In: F Dallmeier and J.A. Comiskey (Eds), Forest Biodiversity in North Central and South America, and the Caribbean: Research and Monitoring. New York: man and biosphere Series, Vol. 21. UNESCO, p. 187-212.

Stiles, G; Skutch, A. 1989. A guide to the birds of Costa Rica.Editorial Cornell University Press.New Jersey, US. 480 p.

Thomlinson, JR, Serran, MI, Del M Lopez, T, Aide, TM, Zimmerman, JK. 1996. Land use dynamic in a post agriculture Puerto Rican landscape (1936-1988). Biotropica, 28: p. 525-536. 